\title{
What happens to unrestored carious deciduous teeth?
}

\author{
The fate of 1,587 unrestored carious deciduous teeth: a retrospective general dental practice based study from \\ northern England R. S. Levine, N. B. Pitts and Z. J. Nugent Br Dent J 2002; 193: 99-103
}

\section{Objective}

To investigate the outcome of non-restoration of carious deciduous teeth by means of a retrospective analysis of clinical case notes of children regularly attending two general dental practices and receiving preventive care.

\section{Design}

Using a carefully defined protocol the fate of deciduous teeth diagnosed as carious into dentine but symptomless and left unrestored was determined from the sequential examination of the clinical records of 481 children attending at least annually.

\section{Results}

The age at initial diagnosis of carious teeth ranged from 1-12 years with the majority of cavities $(1,005)$ presenting by 6 years of age. In all, 1,587 teeth were followed until loss from the mouth. Of these, $190(12 \%)$ were extracted because of pain and a further 60 (4\%) became painful and were treated, leaving 1,337 (84\%) that remained symptomless until being lost. Of the 1,337 symptomless teeth, 178 were extracted under general anaesthesia at the same time as painful ones. The final group of 1,159 (74\%) teeth were exfoliated without causing pain after a mean survival time of 1,332 days. Excluding from the analysis the 178 extracted, but symptomless teeth, leaves a total of 1,409 teeth of which $18 \%$ gave pain and were extracted or treated and the remaining $82 \%$ exfoliated. The strongest determinant of pain was age on diagnosis, the other factors being tooth type and extent of the cavity when first seen. The carious teeth most likely to cause symptoms were found to be molars that developed cavities with pulpal involvement by the age of 3 years, 34\% of which caused pain. In contrast, those least likely to cause pain were carious molar teeth presenting without pulpal involvement after 8 years, only $6 \%$ of which produced symptoms.

\section{Conclusion}

In these patients, the majority of unrestored carious deciduous teeth remain symptomless until shed. The results provide evidence to aid the treatment planning of carious deciduous teeth in children regularly receiving regular preventive dental care.

\section{IN BRIEF}

- Retrospective data on the outcome of non-restoration of carious deciduous teeth.

- The development of pain is related to age at diagnosis, cavity type and tooth type.

- The majority of carious deciduous teeth remained pain-free until exfoliated.

- The study may provide an aid to treatment planning.

\section{COMMENT}

The appearence of this study is timely following the recent publication of the findings from similar studies ${ }^{1-4}$ in the north west of England and the ensuing debate in the letters page of the BDJ. This study reported the outcomes of unrestored carious primary teeth in 481 children attending two dental practices in Leeds and Halifax. The principal finding was that over $80 \%$ of carious unrestored primary teeth remained symptomless until natural exfoliation. Pain was significantly more likely to occur if caries was first recorded at a very young age. Pain was also more likely in molar teeth and teeth with multi-surface lesions. Perhaps the most surprising findings were that only $34 \%$ of the teeth considered to have the worst prognosis (primary molars with pulpal involvement at the age of three) caused pain, and that $60.4 \%$ of teeth with pulpal exposure painlessly exfoliated. If the 70 teeth that had no pain but were extracted were excluded, 74.6 $\%$ of teeth with pulpal exposure exfoliated without pain.

This study looked at the outcomes of the care provided by one dentist, yet they correspond closely with the findings of a pilot study involving four dentists ${ }^{1}$ and a larger study of 50 dentists. ${ }^{2-4}$

In the studies reported by our team there was no difference in the outcomes of restored and unrestored primary teeth, and although there was a variation in the mean percentage of teeth filled per child by the dentists, the majority of carious primary teeth were filled (40.5\% incisors and canines, $81.1 \%$ first molars, $84.3 \%$ second molars). ${ }^{3}$ It is perhaps unfortunate that Levine etal. could not present data for restored carious primary teeth, however similar results were achieved by eschewing a restorative approach and adopting a preventive model of care.

Both the study by Levine et al. and our analyses found the same significant relationships between adverse outcomes and tooth type, lesion size and the age when caries was first recorded. The results of these studies tell similar stories and increase confidence in the validity of the findings. These studies should force the profession to re-evaluate the nature of dental caries in the primary dentition. If approximately $80 \%$ of carious teeth exfoliate naturally without pain; just how big a public health problem is the disease in the primary dentition, and can costly, ${ }^{5}$ possibly traumatic clinical interventions be necessarily justified? Further research is needed to answer these fundamental questions if the profession is to maintain its commitment to evidence-based policy and practice.

Dr Martin Tickle, Consultant|Senior Lecturer in Dental Public Health, Manchester University Dental School

1. Tickle M, Milsom K M, Kennedy A. Is it better to leave or restore carious deciduous molar teeth? A preliminary study Prim Dent Care 1999: 6; 127-131.

2. Milsom KM, Tickle M, King D, Kearney-Mitchell P, Blinkhorn AS. Outcomes associated with restored and unrestored deciduous molar teeth Prim Dent Care 2002; 9: 16-19.

3. Tickle M, Milsom K M, King D, Kearney-Mitchell P, Blinkhorn AS. The fate of the carious primary teeth of children who regularly attend the general dental service BrDentJ 2002; 192: 219-223.

4. Milsom K M, Tickle M, Blinkhorn A S. Dental pain and dental treatment of young children attending the general dental service BrDentJ 2002; 192: 280-284.

5. Shelley A. Mackie I. Case Study of an anxious child with extensive caries treated in general practice: financial viability under the terms of the UK National Health Service. Dent Update 2001; 28: 418-423. 